\title{
Differences Between Post-Authorization Adverse Drug Reactions of Biopharmaceuticals and Small Molecules
}

\author{
Hans C. Ebbers • Esraa Al-Temimi • \\ Ellen H. M. Moors • Aukje K. Mantel-Teeuwisse • \\ Huub Schellekens $\cdot$ Hubert G. M. Leufkens
}

Published online: 12 February 2013

(c) Springer International Publishing Switzerland 2013

\begin{abstract}
Background The nature of adverse drug reactions observed post-authorization for biopharmaceuticals differs from that observed for chemically synthesized, small molecules (SMs). However, it remains unclear how much of the observed differences can be attributed to differences in authorized indications of the two product groups.

Objective To investigate if the nature of adverse drug reactions identified post-authorization for biopharmaceuticals differs from those of SMs within the same anatomical therapeutic chemical (ATC) group.
\end{abstract}

Electronic supplementary material The online version of this article (doi:10.1007/s40259-013-0012-y) contains supplementary material, which is available to authorized users.

H. C. Ebbers - E. Al-Temimi - A. K. Mantel-Teeuwisse ( $\square)$.

H. G. M. Leufkens

Division of Pharmacoepidemiology and Clinical Pharmacology, Faculty of Science, Utrecht Institute for Pharmaceutical

Sciences (UIPS), Utrecht University, P.O. Box 80 082,

3508 TB Utrecht, The Netherlands

e-mail: A.K.Mantel@uu.nl

E. H. M. Moors - H. Schellekens

Copernicus Institute/Department of Innovation and

Environmental Studies, Utrecht University, Utrecht,

The Netherlands

H. Schellekens

Department of Pharmaceutics, Faculty of Science, Utrecht Institute for Pharmaceutical Sciences (UIPS),

Utrecht, The Netherlands

H. G. M. Leufkens

Medicines Evaluation Board, Utrecht, The Netherlands
Methods We analyzed safety issues included in postauthorization, changes to the Summary of Product Characteristics of centrally approved products in the European Union classified in the ATC main group of 'antineoplastic and immunomodulating agents'. Generics and biosimilars were excluded. All issues identified during 2004-2011 were analyzed for differences in nature and timing between biopharmaceutical and SM products, at different ATC levels.

Results A total of 747 adverse drug reactions were identified; 361 for biopharmaceuticals and 386 for SMs. Within the sub group of immunosuppressants, neoplasms (20\% vs $2 \%, p<0.01)$ and infections and infestations ( $22 \%$ vs $9 \%$, $p<0.01$ ) occurred significantly more frequent for biopharmaceuticals. Adverse drug reactions of SMs were more often renal and urinary disorders (7\% vs $0 \%, p<0.01$ ), blood and lymphatic system disorders $(10 \%$ vs $3 \%$, $p=0.04)$, and vascular disorders ( $7 \%$ vs $1 \%, \mathrm{p}=0.02)$. In the subgroup of antineoplastics, immune system disorders occurred more frequently for biopharmaceuticals, $(6 \%$ vs $1 \%, p=0.04)$. With the exception of immune system disorders and renal disorders, the overall differences between biopharmaceuticals and SMs were mostly caused by products authorized as immunosuppressants. For the subset of products authorized after 2004, the median time to the first safety issue was 18 months (95\% CI 12.4-21.5) for biopharmaceuticals and 17 months (95\% CI 12.5-21.5) for SMs and did not differ significantly within subgroups.

Conclusion Even within a group of medicinal products approved in the same indication, differences were observed in the nature of adverse drug reactions between biopharmaceuticals and SMs. The considerable differences in the nature of adverse drug reactions between biopharmaceuticals and SMs were not associated with differences in the timing of regulatory actions. 


\section{Background}

A stable number of biopharmaceuticals has entered the clinic over the past years. Biopharmaceuticals are here defined as therapeutic (glyco)proteins produced using biotechnology. They differ from chemically synthesized entities (small molecules, SMs) in various ways including size, structure, homogeneity, stability and immunogenic potential, which may lead to a specific adverse profile for biopharmaceuticals $[1,2]$. It has been suggested that the unique safety risks associated with biopharmaceuticals may require a tailored pharmacovigilance strategy to identify and minimize risks associated with their use [3]. Earlier research has shown that spontaneously reported adverse drug reactions reported post-approval for biopharmaceuticals differ from those reported for SMs [4]. In addition, the nature and sources of post-approval safety issues requiring regulatory actions differ between biopharmaceuticals and SMs [5, 6]. For example, it has been found that the main post-approval safety warnings of biopharmaceuticals concern infections and neoplasms [5]. Furthermore, it has been suggested that the timing of regulatory actions may differ for SMs and biopharmaceuticals [5, 7]. Although the observed differences may hold on an aggregated level, there are several factors that may contribute to these observed differences that require further clarification.

First, significant differences exist in approved indications between biopharmaceuticals and SMs. For example, biopharmaceuticals often belong to the anatomical therapeutic chemical (ATC) main groups of 'antineoplastic and immunomodulating agents', 'alimentary tract and metabolism' and 'blood and blood forming organs', whereas SMs often belong to 'anti-infectives for systemic use', 'antineoplastic and immunomodulating agents' and 'nervous system drugs' [8]. The underlying disease may affect the nature of adverse drug reactions for a given product and an imbalance in authorized indications may explain much of the observed difference in safety profiles. Second, within a single indication, biopharmaceuticals are often prescribed to different patient populations than SMs, which may have different susceptibilities to develop adverse drug reactions. For example, biopharmaceuticals are often recommended as second- or third-line products for patients who have failed to respond to at least one non-biopharmaceutical systemic treatment, as in rheumatoid arthritis and psoriasis $[9,10]$. Rheumatoid arthritis patients are at an increased risk of infections both by their immunosuppressive therapies as well as the disease itself [11]. Likewise, psoriasis patients often receive systemic medication and/or light therapy that may predispose them to specific neoplasms [12]. Third, the adverse drug reactions may be the result of intrinsic properties of the molecules. Many biopharmaceuticals are immunosuppressants and their efficacy is associated with the level of immunosuppression and therefore with a risk of infections and various cancers [13]. The relative importance of these factors for the observed differences in the nature of adverse drug reaction profiles for biopharmaceuticals remains unclear. In an attempt to further elucidate this we have analyzed postapproval safety events within the same patient population [14]. Furthermore, we have assessed whether these differences affect the timing of regulatory actions. As most of the currently approved biopharmaceuticals are authorized as 'antineoplastic and immunomodulating agents', our study was performed in this group of products $[8,15]$.

\section{Methods}

We have analyzed all safety issues included in post-approval regulatory actions taken for SMs and biopharmaceuticals that belong to the anatomic therapeutic main group of 'antineoplastic and immunomodulating agents' ('L'), centrally approved in the European Union between 1995 and 1 July 2011. We will refer to this selection as 'group' throughout the remainder of the manuscript [14]. Generic products and biosimilars were excluded, as well as identical products sold under different trade names and products that were withdrawn without publishing safety data. Products were further subdivided into the ATC therapeutic subgroups L01 'antineoplastics', L02 'endocrine therapy', L03 'immunostimulants', and L04 'immunosuppressants'.

Safety issues were identified from post-approval adaptations of the European Summary of Product Characteristics (SPC) through so-called 'type II variations'. Type II variations are recorded in European Public Assessment Reports (EPARs) that are publicly available through the website of the European Medicines Agency [16]. As detailed information on the nature and timing of type II variations is only available from 2004, we only included safety issues that occurred from 2004 onwards.

Both newly identified (potential) safety issues and additional information on known safety events leading to new type II variations were included. All variations that were part of extensions of the indication, or variations not providing new safety information were excluded; for example, the addition of data on pediatric dosing schedules. The nature of each safety event was identified and coded according to MedDRA (Medical Dictionary for Regulatory Activities) v. 14.1 (UK) and classified according to system organ class (SOC). If no corresponding MedDRA term existed, an equivalent term was selected by consensus (Ebbers and Al-Temimi).

All results were coded, entered into a database, and analyzed using SPSS v. 190 (SPSS, Inc., Chicago, IL, USA). Differences between non-normally distributed data 
were tested using the Wilcoxon Mann-Whitney test. Differences between requirements for SPC changes and determinants, including product characteristics, regulatory authorization characteristics, and time since approval, were tested using two-sided Fisher's exact tests at an $\alpha$-level of 0.05 . In order to assess the time to first regulatory action, survival curves were created for the subset of products that were approved from 2004. The median time to first regulatory action for each group was calculated using KaplanMeier methods.

\section{Results}

We identified 127 products that were approved in the group of antineoplastic and immunomodulating agents, of which 36 were excluded leaving a total of 91 products, 33 biopharmaceuticals and 58 SMs (Fig. 1). For 7 biopharmaceuticals and $20 \mathrm{SMs}$, no safety issues were identified. The two groups were comparable with regard of the 'age' of the products. The median time between authorization of a product and the end of the study period was 7.4 years (interquartile range [IQR] 2.5-11.4) for biopharmaceuticals versus 5.0 years (IQR 2.5-10.0) for SMs $(p=0.34)$.

A list of the products included in the study, and the number of safety issues per product, is provided in Annex I (see Online Resource 1). The proportion of products for which at least one safety issue was identified did not differ significantly between biopharmaceuticals and SMs; 26 $(79 \%)$ versus $38(66 \%)(p=0.24)$. The final sample included 362 type II variations describing 747 individual safety issues; 361 for biopharmaceuticals and 386 for SMs (Table 1). For SM products classified as 'antineoplastics', protein kinase inhibitors accounted for the majority (157 [92\%]) of the safety issues. Bevacizumab (Avastin ${ }^{\circledR}$ ) accounted for the majority (35 [56\%]) of the safety issues reported for biopharmaceuticals in this subgroup. In the subgroup of immunosuppressants (L04), 119 (70\%), of the safety issues of biopharmaceuticals originated from tumor necrosis factor inhibitors (TNFIs).

\subsection{Nature of Safety Events}

Overall, safety issues included in post-approval safetyrelated regulatory actions for biopharmaceuticals were classified most often as infections and infestations (14.1\%) and neoplasms (10.5\%), while for SMs they were most often classified as general disorders and administration site conditions $(15.8 \%)$ and skin and subcutaneous disorders (11.7\%) (Fig. 2). There were five SOCs that showed a consistent difference in both subgroups: infections and infestations, skin and subcutaneous disorders, neoplasms, immune system disorders, and renal and urinary disorders.

The differences between biopharmaceuticals and SMs were most pronounced in the subgroup of immunosuppressants (Table 2). The majority of 38 infection-related
Fig. 1 Flowchart of safety issues included in the final analysis

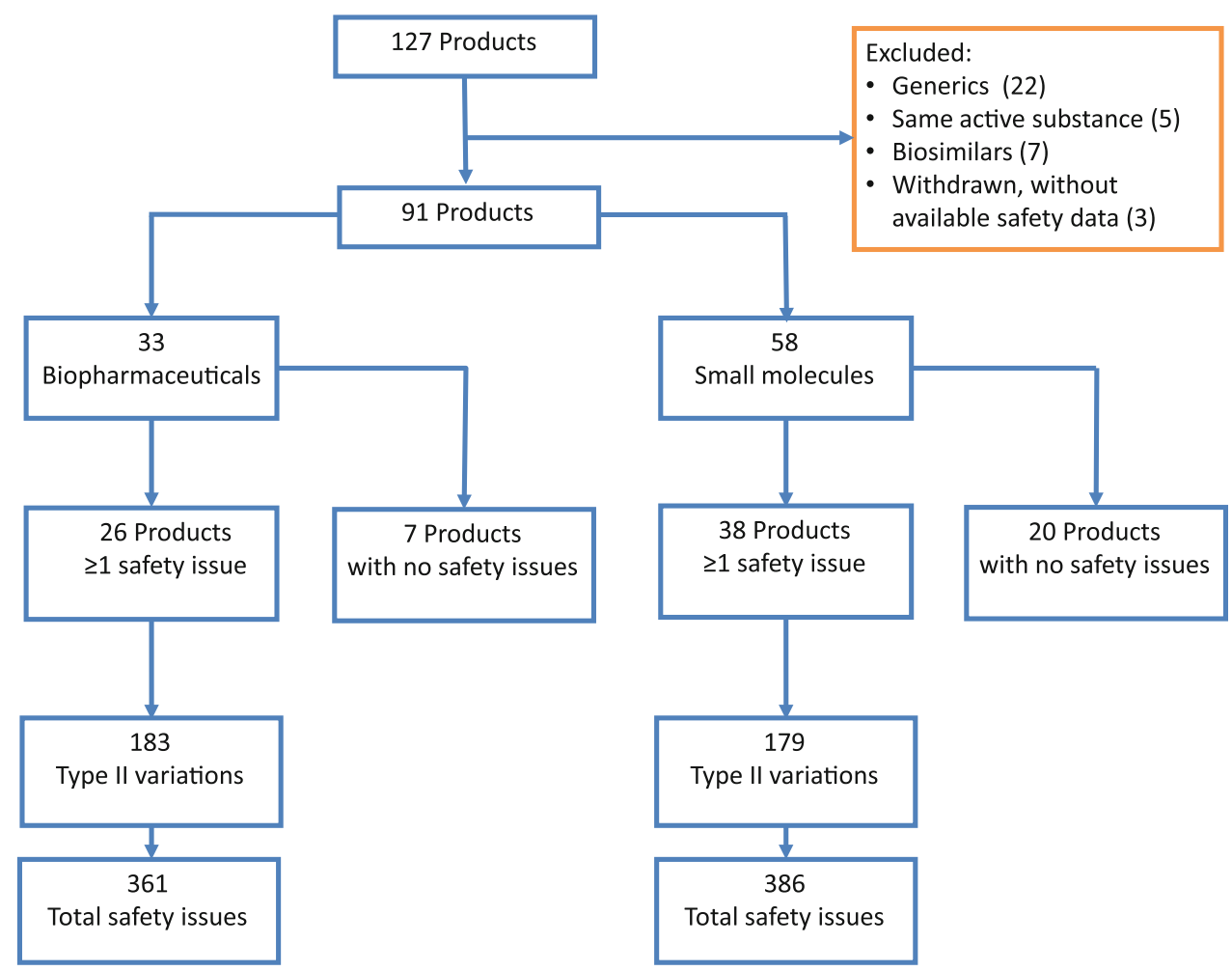


Table 1 Products, type II variations and safety issues included for products approved within the ATC main group of immunosuppressants and immunomodulators per ATC subgroup

\begin{tabular}{|c|c|c|c|c|c|c|}
\hline \multirow[t]{2}{*}{ ATC subgroup } & \multicolumn{2}{|l|}{ Products $^{\mathrm{a}}$} & \multicolumn{2}{|l|}{ Type II variations } & \multicolumn{2}{|l|}{ Safety issues } \\
\hline & Biopharmaceutical & SM & Biopharmaceutical & $\mathrm{SM}$ & Biopharmaceutical & SM \\
\hline L01 antineoplastics & $6(2)$ & $30(13)$ & 29 & 129 & $62(17 \%)$ & $288(75 \%)$ \\
\hline L02 endocrine therapy & 0 & $2(1)$ & 0 & 4 & $0(0 \%)$ & $6(2 \%)$ \\
\hline L03 immunostimulants & $7(2)$ & $0(4)$ & 59 & 0 & $128(36 \%)$ & $0(0 \%)$ \\
\hline L04 immunosuppressants & $13(4)$ & $6(3)$ & 95 & 46 & $171(47 \%)$ & $92(24 \%)$ \\
\hline Total & $26(7)$ & $38(20)$ & 183 & 179 & $361(100 \%)$ & $386(100 \%)$ \\
\hline
\end{tabular}

ATC anatomical therapeutic chemical, $S M$ small molecules

a The numbers in parentheses include products with no safety concerns

Fig. 2 Proportions of regulatory actions per system organ class $(S O C)$. SOCs were only included in the graph if the combined frequency of safety issues was $\geq 5 \%$. The remaining SOCs were included in a single category: 'other' $* p<0.05$ for difference between biopharmaceuticals and small molecules
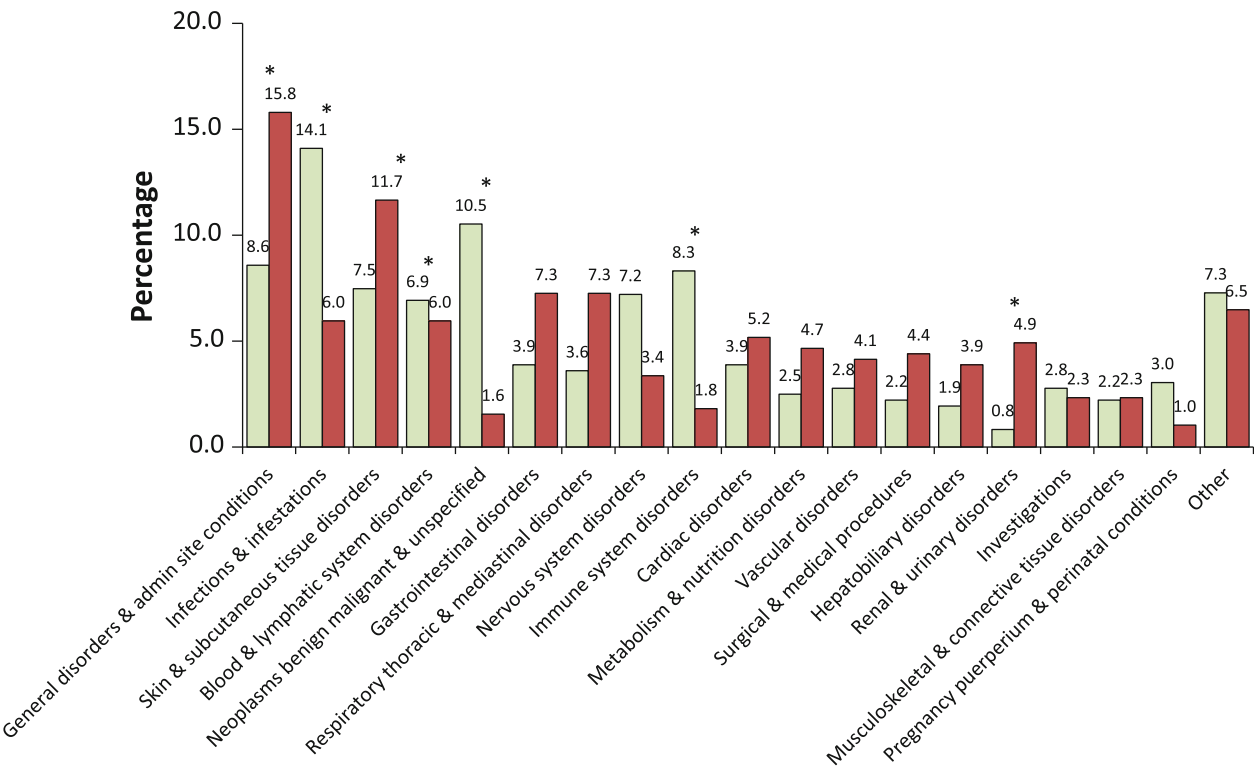

Small molecules $(n=386)$ post-approval safety issues were issued for TNFIs ( $n=22$; $58 \%$ ). Of the seven infections included in products classified as antineoplastics, four were issued for rituximab which is also indicated for the treatment of rheumatoid arthritis. Similarly, of the 35 safety issues classified as neoplasms, 31 (85\%) originated from TNFIs, of which 10 resulted from a class review that added additional warnings to all five authorized TNFIs related to a possible increased risk of leukemia and pediatric malignancies. In a sensitivity analysis we included only the first in class TNFI (etanercept) and excluded all other TNFIs, leaving 74 safety issues for biopharmaceuticals. Both the proportions of safety issues classified as infections (24.3\% versus $8.7 \%$; $p<0.01)$ and neoplasms (13.8\% versus $2.9 \% ; p=0.012)$ were still significantly higher for biopharmaceuticals than for SMs. For antineoplastics, fewer differences were observed, with the exception of the relatively large proportion of 'general disorders and administration site conditions' for SMs (18\%). Of the 53 issues included in this category, 35 (66\%) were interactions with other drugs, while for biopharmaceuticals classified as antineoplastic, the category 'general disorders' included mostly infusion reactions. Safety issues classified as renal disorders occurred mostly for SMs. The only case of renal and urinary disorders for the group of biopharmaceuticals was reported for panitumumab. All but one case within the SOC surgical and medical procedures concerned SMs, most of which related to dose adjustments and changes to treatment regimens, the majority of which related to patients with renal or hepatic impairment. Within the group of safety concerns that occurred less frequently than $5 \%$, significantly more safety issues were classified as 'eye disorders' for biopharmaceuticals $(10.7 \%$ versus $0.3 \% ; p<0.001)$. All these issues were included in a single Type II variation, 
Table 2 Frequency of safety issues classified per system organ class level per ATC sub group

\begin{tabular}{|c|c|c|c|c|c|c|c|c|c|c|}
\hline \multirow[t]{3}{*}{ SOC } & \multicolumn{5}{|c|}{ L01 (antineoplastics) } & \multicolumn{5}{|c|}{ L04 (Immunosuppressants) } \\
\hline & \multicolumn{2}{|c|}{ Biopharmaceuticals } & \multicolumn{2}{|c|}{$\begin{array}{l}\text { Small } \\
\text { molecules }\end{array}$} & \multirow[t]{2}{*}{$p$ value } & \multicolumn{2}{|c|}{ Biopharmaceuticals } & \multicolumn{2}{|c|}{$\begin{array}{l}\text { Small } \\
\text { molecules }\end{array}$} & \multirow[t]{2}{*}{$p$ value } \\
\hline & $\mathrm{n}$ & $\%$ & $\mathrm{n}$ & $\%$ & & $\mathrm{n}$ & $\%$ & $\mathrm{n}$ & $\%$ & \\
\hline General disorders and administration site conditions & 4 & 6.5 & 53 & 18.4 & 0.022 & 12 & 7.0 & 5 & 5.4 & 0.794 \\
\hline Infections and infestations & 7 & 11.3 & 15 & 5.2 & 0.085 & 38 & 22.2 & 8 & 8.7 & 0.006 \\
\hline Skin and subcutaneous tissue disorders & 2 & 3.2 & 34 & 11.8 & 0.062 & 15 & 8.8 & 11 & 12.0 & 0.516 \\
\hline Blood and lymphatic system disorders & 6 & 9.7 & 14 & 4.9 & 0.139 & 5 & 2.9 & 9 & 9.8 & 0.039 \\
\hline Neoplasms benign, malignant and unspecified & 3 & 4.8 & 4 & 1.4 & 0.109 & 35 & 20.5 & 2 & 2.2 & $<0.001$ \\
\hline Gastrointestinal disorders & 5 & 8.1 & 21 & 7.3 & 0.792 & 4 & 2.3 & 7 & 7.6 & 0.054 \\
\hline Respiratory, thoracic and mediastinal disorders & 5 & 8.1 & 22 & 7.6 & 1.000 & 6 & 3.5 & 6 & 6.5 & 0.353 \\
\hline Nervous system disorders & 2 & 3.2 & 8 & 2.8 & 0.693 & 11 & 6.4 & 5 & 5.4 & 1.000 \\
\hline Immune system disorders & 4 & 6.5 & 4 & 1.4 & 0.036 & 17 & 9.9 & 3 & 3.3 & 0.054 \\
\hline Cardiac disorders & 5 & 8.1 & 17 & 5.9 & 0.563 & 4 & 2.3 & 3 & 3.3 & 0.698 \\
\hline Metabolism and nutrition disorders & 3 & 4.8 & 13 & 4.5 & 1.000 & 2 & 1.2 & 5 & 5.4 & 0.053 \\
\hline Vascular disorders & 4 & 6.5 & 10 & 3.5 & 0.284 & 2 & 1.2 & 6 & 6.5 & 0.023 \\
\hline Surgical and medical procedures & 0 & 0.0 & 12 & 4.2 & 0.136 & 1 & 0.6 & 4 & 4.3 & 0.052 \\
\hline Hepatobiliary disorders & 0 & 0.0 & 15 & 5.2 & 0.083 & 4 & 2.3 & 0 & 0.0 & 0.301 \\
\hline Renal and urinary disorders & 1 & 1.6 & 13 & 4.5 & 0.479 & 0 & 0.0 & 6 & 6.5 & 0.002 \\
\hline
\end{tabular}

Only safety issues that occurred at a frequency of $>5 \%$ of the overall sample are included in the table

$A T C$ anatomical therapeutic chemical, SOC system organ class

which related to the off-label use of bevacizumab in agerelated macular degeneration.

\subsection{Timing of Post-Approval Regulatory Actions}

For the subset of products that were approved from 1 January 2004, we performed a survival analysis to determine the time to first regulatory action. Of 16 biopharmaceuticals included, $11(69 \%)$ had one or more post-approval safety issue, compared with $23(62 \%)$ of the SMs. The median time to the first safety issue was 18 months (95\% CI 12.4-21.5) for biopharmaceuticals and 17 months (95 \% CI 12.5-21.5) for SMs $(p=0.88)$ (Fig. 3). Analyses within subgroups yielded similar results (data not shown). Altogether, these data do not imply a difference in the time until the first regulatory action between biopharmaceuticals and SMs within the same therapeutic class.

\section{Discussion}

We have compared the nature of post-approval safety issues for biopharmaceuticals and SMs, for a group of products within the same therapeutic group. The great majority of products had at least one post-approval safety issue. Overall, we observed substantial differences between

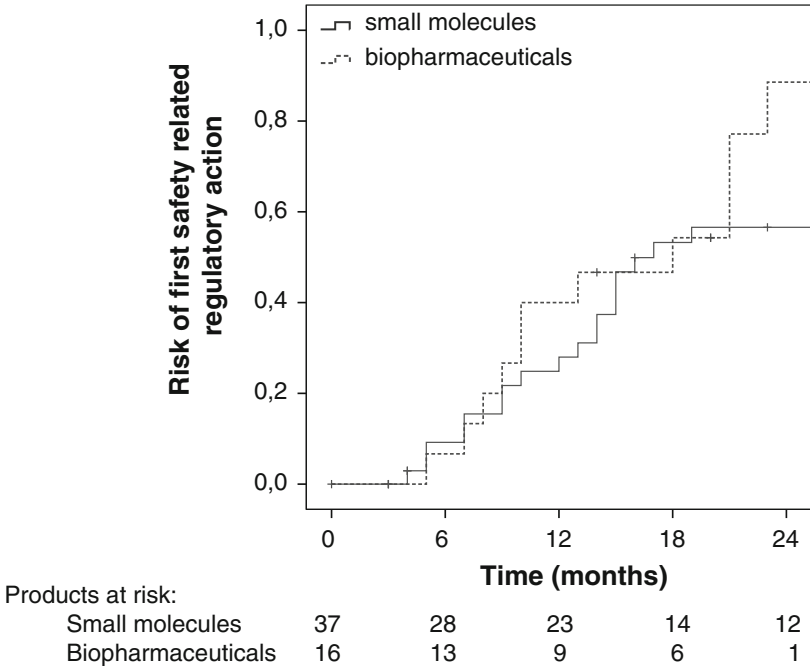

Fig. 3 Time to first regulatory action for biopharmaceuticals and small molecules

biopharmaceuticals and SMs. Safety issues classified as immune system disorders occurred more frequently in both antineoplastics and immunosuppressants (although only significantly different for immunosuppressants), whereas renal disorders occurred less frequently for biopharmaceuticals than for SMs. The subgroup of immunosuppressants accounted mostly for the observed increase in the frequency of infections and infestations and neoplasms. 
The differences observed in the nature of post-authorization safety issues between biopharmaceuticals and SMs was not associated with obvious differences in the outcome of the pharmacovigilance process as determined by the time to first regulatory action.

Our analysis was performed in the same therapeutic class, and anatomic therapeutic subgroups (i.e., antineoplastics and immunosuppressants), whereas previous studies analyzed differences between biopharmaceuticals and SMs without making this distinction. In addition, we analyzed all individual safety concerns included for these products amounting to a substantial number $(n=747)$ of safety issues, which allowed for a comparison between the two groups. The fact that we found an increase in infections and neoplasms for immunosuppressants, but not in antineoplastics, suggests that immunosuppressants accounted for many of the differences observed in earlier studies. Indeed, 7 of 11 Direct Healthcare Professional Communications issued for biologics between 1995 and 2007 were issued for immunosuppressants and the majority of individual case reports reported for biologics were reported for immunosuppressants (mainly TNFIs) [4, 5]. A possible explanation may be the strong pharmacological actions of many biopharmaceutical immunosuppressants, which is also reflected in their efficacy in treating autoimmune diseases. The risk of cancer may be further increased by the fact that patients receiving a biopharmaceutical immunosuppressant have a longer history of, often poorly controlled, disease [17]. The increase in neoplasms and infections may be attributed to the mechanism of action of the products included in the study and, as such, categorized as pharmacologically mediated events [1].

Unlike earlier studies, our study found a relatively large contribution of safety issues classified as 'general disorders and administration site conditions' for SMs, especially for the groups of antineoplastic agents $[4,5]$. This may be explained by type II variations that included data on interactions, which agrees with what has been reported previously [7]. As interactions are often identified through pharmacokinetic/pharmacodynamic studies, they may not show up in spontaneous reporting databases [4]. Our results show an increase in immune system disorders, which was apparent in both sub-groups. This is consistent with the immunogenic potential of most biopharmaceuticals. SMs demonstrated significantly more safety issues concerning renal and urinary disorders, which may be explained by the fact that, unlike biopharmaceuticals, SMs may form active metabolites. Our study included one safety issue for a biopharmaceutical classified as a renal disorder, namely cases of renal failure seen in patients receiving panitumumab (Vectibix ${ }^{\circledR}$ ), which was related to the product's pharmacological mechanism [18]. All in all, our results are in line with the view that adverse drug reactions of biopharmaceuticals are only the result of their pharmacological actions and immunogenicity.

Interestingly, differences in the nature of post-approval safety issues were less pronounced for antineoplastics than for immunosuppressants. Antineoplastics are mostly products that are used in the first line (e.g., trastuzumab, rituximab, panitumumab, or bevacizumab). It is therefore possible that, within the subgroup of antineoplastics, patients receiving biopharmaceuticals and SMs are more comparable than within the immunosuppressant subgroup. Furthermore, antineoplastic biopharmaceuticals are usually given in combination with chemotherapy, whereas SMs were prescribed mainly as monotherapy. Future research could focus on characteristics of safety issues in other therapeutic indications, in which similar patients are treated with biopharmaceuticals and SMs.

We observed no differences in timing to first regulatory action between biopharmaceuticals and SMs. Mol et al. [6] reported that Direct Healthcare Professional Communications (DHPCs) for biopharmaceuticals were issued earlier than for SMs. However, they identified DHPCs and retrospectively assessed the time to the authorization date, which amounted to several decades for a number of SM products. It is thus likely that the difference they observed is due to 'age differences' between the groups, that is, the average age of the SMs at risk at the beginning of their study is likely to be much larger than for biopharmaceuticals. Also, Giezen et al. did not find significant differences between the timing to adverse drug reactions as compared with earlier studies including mostly SMs [5]. These results do not point to an increased risk for postapproval regulatory actions of biopharmaceuticals.

Several limitations apply to our study, which may have influenced the outcome. Firstly, the anatomic therapeutic subgroups were dominated by a limited number of products; for example, biopharmaceuticals in the ATC subgroup of immunosuppressants were mainly TNFIs, and bevacizumab $\left(\right.$ Avastin $\left.^{\circledR}\right)$ accounted for half of the safety issues included in the antineoplastics category. Currently there are five TNFIs authorized in the EU, a single class event could have a disproportionate influence on the overall result. Nevertheless, a sensitivity analysis excluding all but the first in class TNFI did not lead to changes in the overall outcome. Secondly, we based our analysis on EPARs. Although EPARs are an adequate source for the identification of safety issues, it was sometimes challenging to find corresponding MedDRA terms. Thirdly, in the current study we were not able to control for possible differences in patient populations, such as disease duration, disease severity, or medication history. Finally, we did not have access to data on patient exposure of the products included in the study. Therefore, we are unable to conclude on the relative incidence of adverse drug reactions occurring for biopharmaceuticals and SMs. 
Nevertheless, it is clear that some products are much more widely prescribed than others, which may partially be reflected in the number of safety issues that were identified post-authorization.

The question remains what our results mean for the safety management of biopharmaceuticals. A key reason to come to a valid classification of adverse drug reactions is to optimize pharmacovigilance planning, so that the benefitrisk profile of products entering the market is established quickly and rationally [19]. Historically, adverse drug reactions have been classified as Type A or Type B reactions, where Type A reactions have been defined as pharmacology related, dose-dependent events and Type B as idiosyncratic, often immune-related events [20]. This classification has several limitations and is increasingly criticized for multiple reasons that have been discussed elsewhere [21]. Another mechanistic classification system has been proposed to classify adverse drug effects [22]. Yet another classification system has been developed for biopharmaceuticals, which focuses mainly on immunological reactions [23]. While these classifications may be instrumental in describing adverse reactions, they may not be optimal to guide pharmacovigilance efforts. Shankar et al. proposed a classification system that categorizes products based on the risks of immunogenicity-associated adverse drug reactions [24]. This classification may be instrumental in identifying immunogenicity-associated events and can serve as guidance for targeted pharmacovigilance strategies. While it is important to gain insight into how immunological reactions give rise to post-authorization safety concerns, our results indicate a larger fraction of post-authorization adverse drug reactions is associated with the intended mechanism of action of a product. At the time of authorization, knowledge of the adverse outcomes of hitting a certain target is limited. A thorough understanding of the biology of the target is needed and past experience may guide risk management activities; e.g., activities to establish possible increases in malignancies and/or infections for biopharmaceutical immunosuppressants.

As biopharmaceuticals are mostly prescribed in a hospital setting, various widely used epidemiological data sources to assess adverse events post-authorization may have limited applicability and new data sources such as registries may need to be set up [3]. The benefit-risk profile of biopharmaceutical immunosuppressants has, for example, been extensively studied in such post-authorization registries [25]. Registries are especially suitable to assess rare events and events with a long latency such as neoplasms and opportunistic infections like progressive multifocal encephalopathy. However, a considerable number of safety concerns included events that may not have been expected at the time of authorization, underscoring the continued importance of spontaneous reporting systems to monitor unexpected adverse events. This is in line with the results of a previous study that found that, in a limited sample of products for which risk management plans were submitted, half of all post-authorization regulatory actions were included in the risk management plan, while the other half resulted from activities outside the plan, supporting the need for continuous monitoring for unexpected events [26]. Despite significant differences in the nature of postauthorization safety concerns, our results do not indicate a different outcome for combined pharmacovigilance efforts.

\section{Conclusion}

Here we show that post-approval safety issues observed for biopharmaceuticals differ from those observed in SMs, even for products within the same therapeutic class. The considerable difference in the nature of adverse drug reactions between biopharmaceuticals and SMs was not associated with differences in the timing of regulatory actions. Immunological reactions occurred more frequently for biopharmaceuticals and specific pharmacovigilance strategies should be designed to optimally address them. A thorough understanding of the target biology and the intended mechanism of action of the product is most important to guide pharmacovigilance activities of biopharmaceuticals.

Acknowledgments This study was performed in the context of the Escher project (T6-202), a project of the Dutch Top Institute Pharma. All authors declare no conflicts of interest relevant to the subject matter in this manuscript. The Department of Pharmacoepidemiology and Clinical Pharmacology, Utrecht Institute for Pharmaceutical Sciences, employing authors Hans C. Ebbers, Aukje K. Mantel-Teeuwisse, and Hubert G.M. Leufkens, has received unrestricted research funding from the Netherlands Organisation for Health Research and Development (ZonMW), the Dutch Health Care Insurance Board (CVZ), the Royal Dutch Pharmacists Association (KNMP), the private-public funded Top Institute Pharma (www.tipharma.nl, including co-funding from universities, government, and industry), the EU Innovative Medicines Initiative (IMI), EU 7th Framework Program (FP7), the Dutch Medicines Evaluation Board, the Dutch Ministry of Health, and industry (including GlaxoSmithKline, Pfizer, and others). H. Schellekens participated in meetings and publications sponsored by Amgen, J\&J, Roche, Sandoz, and Hospira. Part of his research is directly or indirectly sponsored by Roche and Amgen.

\section{References}

1. Clark JB. Mechanisms of adverse drug reactions to biologics. In: Uetrecht J, editor. Adverse drug reactions. Berlin: Springer; 2010. p. $453-74$.

2. Baumann A. Early development of therapeutic biologics: pharmacokinetics. Curr Drug Metab. 2006;7(7):15-21.

3. Giezen TJ, Mantel-Teeuwisse AK, Leufkens HG. Pharmacovigilance of biopharmaceuticals: challenges remain. Drug Saf. 2009; 32(10):811-7. 
4. Giezen TJ, Mantel-Teeuwisse AK, Meyboom RH, Straus SM, Leufkens HG, Egberts TC. Mapping the safety profile of biologicals: a disproportionality analysis using the WHO adverse drug reaction database, VigiBase. Drug Saf. 2010;33(10):865-78.

5. Giezen TJ, Mantel-Teeuwisse AK, Straus SM, Schellekens H, Leufkens HG, Egberts AC. Safety-related regulatory actions for biologicals approved in the United States and the European Union. JAMA. 2008;300(16):1887-96.

6. Mol PG, Straus SM, Piening S, de Vries JT, de Graeff PA, Haaijer-Ruskamp FM. A decade of safety-related regulatory action in the Netherlands: a retrospective analysis of direct healthcare professional communications from 1999 to 2009. Drug Saf. 2010;33(6):463-74.

7. Lasser KE, Allen PD, Woolhandler SJ, Himmelstein DU, Wolfe SM, Bor DH. Timing of new black box warnings and withdrawals for prescription medications. JAMA. 2002;287(17):2215-20.

8. Motola D, De Ponti F, Poluzzi E, Martini N, Rossi P, Silvani MC, et al. An update on the first decade of the European centralized procedure: how many innovative drugs? $\mathrm{Br} \mathrm{J}$ Clin Pharmacol. 2006;62(5):610-6.

9. Smolen JS, Landewe R, Breedveld FC, Dougados M, Emery P, Gaujoux-Viala C, et al. EULAR recommendations for the management of rheumatoid arthritis with synthetic and biological disease-modifying antirheumatic drugs. Ann Rheum Dis. 2010; 69(6):964-75.

10. Pathirana D, Ormerod AD, Saiag P, Smith C, Spuls PI, Nast A, et al. European S3-guidelines on the systemic treatment of psoriasis vulgaris. J Eur Acad Dermatol Venereol. 2009;23(Suppl. 2): $1-70$.

11. Doran MF, Crowson CS, Pond GR, O'Fallon WM, Gabriel SE. Predictors of infection in rheumatoid arthritis. Arthritis Rheum. 2002;46(9):2294-300.

12. Naldi L. Malignancy concerns with psoriasis treatments using phototherapy, methotrexate, cyclosporin, and biologics: facts and controversies. Clin Dermatol. 2010;28(1):88-92.

13. Vial T, Descotes J. Immunosuppressive drugs and cancer. Toxicology. 2003;185(3):229-40.
14. WHO Collaborating Centre for Drug Statistics Methodology. ATC/DDD Index 2011; 10 May 2011 (online). http://www. whocc.no/atc_ddd_index/. Accessed 18 Jun 2011.

15. Trusheim MR, Aitken ML, Berndt AR. Characterizing markets for biopharmaceutical innovations: do biologics differ from small molecules? Forum Health Econ Pol. 2010;13(1):1-45.

16. European Medicines Agency. EPARs for authorised medicinal products for human use [online]. http://www.ema.europa.eu/ htms/human/epar/a.htm. Accessed 12 Jul 2011.

17. Strangfeld A, Listing J, Herzer P, Liebhaber A, Rockwitz K, Richter C, et al. Risk of herpes zoster in patients with rheumatoid arthritis treated with anti-TNF-alpha agents. JAMA. 2009;301(7): 737-44.

18. Lameire N, Kruse V, Rottey S. Nephrotoxicity of anticancer drugs: an underestimated problem? Acta Clin Belg. 2011;66(5):337-45.

19. Callreus T. Use of the dose, time, susceptibility (DoTS) classification scheme for adverse drug reactions in pharmacovigilance planning. Drug Saf. 2006;29(7):557-66.

20. Aronson JK. Adverse drug reactions: history, terminology, classification, causality, frequency, preventability. Stephens' Detection and evaluation of adverse drug reactions, pp. 1-119. Wiley Ltd, New York (2011).

21. Aronson JK, Ferner RE. Clarification of terminology in drug safety. Drug Saf. 2005;28(10):851-70.

22. Ferner RE, Aronson JK. EIDOS: a mechanistic classification of adverse drug effects. Drug Saf. 2010;33(1):15-23.

23. Pichler WJ. Adverse side-effects to biological agents. Allergy. 2006;61(8):912-20.

24. Shankar G, Pendley C, Stein KE. A risk-based bioanalytical strategy for the assessment of antibody immune responses against biological drugs. Nat Biotechnol. 2007;25(5):555-61.

25. Zink A, Askling J, Dixon WG, Klareskog L, Silman AJ, Symmons DP. European biologicals registers: methodology, selected results and perspectives. Ann Rheum Dis. 2009;68(8):1240-6.

26. Frau S, Font Pous M, Luppino MR, Conforti A. Risk management plans: are they a tool for improving drug safety? Eur J Clin Pharmacol. 2010;66(8):785-90. 